\title{
Quantitative assessment of changes in hemodynamics of the internal carotid artery after bypass surgery for moyamoya disease
}

\author{
Fengping Zhu, MD, PhD, ${ }^{1,2}$ Yi Qian, PhD, ${ }^{2}$ Bin Xu, MD, ${ }^{1}$ Yuxiang Gu, MD, ${ }^{1}$ Kaavya Karunanithi, PhD, ${ }^{2}$ \\ Wei Zhu, MD, ${ }^{1}$ Liang Chen, MD, ${ }^{1}$ Ying Mao, MD, ${ }^{1,3}$ and Michael K. Morgan, MD² \\ 1Department of Neurosurgery, Huashan Hospital; ${ }^{3}$ State Key Laboratory of Medical Neurobiology, School of Basic Medical \\ Sciences and Institutes of Brain Science, Fudan University, Shanghai, China; and ${ }^{2}$ Faculty of Medicine and Health Sciences, \\ Macquarie University, Sydney, New South Wales, Australia
}

\begin{abstract}
OBJECTIVE Although intracranial vessel remodeling has been observed in moyamoya disease, concerns remain regarding the effect of bypass surgery on hemodynamic changes within the internal carotid artery (ICA). The authors aimed to quantify the surgical effect of bypass surgery on bilateral ICAs in moyamoya disease and to estimate pressure drop (PD) along the length of the ICA to predict surgical outcomes.

METHODS Records of patients who underwent bypass surgery for treatment of moyamoya disease and in whom flow rates were obtained pre- and postsurgery by quantitative MR angiography were retrospectively reviewed. Quantitative MR angiography and computational fluid dynamics were applied to measure morphological and hemodynamic changes during pre- and postbypass procedures. The results for vessel diameter, volumetric flow, PD, and mean wall shear stress along the length of the ICA were analyzed. Subgroup analysis was performed for the circle of Willis (CoW) configurations.

RESULTS Twenty-three patients were included. The PD in ICAs on the surgical side (surgical ICAs) decreased by $21.18 \%$ (SD $\pm 30.1 \%$ ) and increased by $11.75 \%$ (SD $\pm 28.6 \%$ ) in ICAs on the nonsurgical side (contralateral ICAs) $(p=$ 0.001). When the PD in contralateral ICAs was compared between patients with a complete or incomplete CoW, the authors found that the PDI in the former group decreased by $2.45 \%$ and increased by $20.88 \%$ in the latter $(p=0.05)$. Regression tests revealed that a greater postoperative decrease in PD corresponded to shrinking of $I C A s\left(R^{2}=0.22, p=\right.$ 0.02).

CONCLUSIONS PD may be used as a reliable biomechanical indicator for the assessment of surgical treatment outcomes. The vessel remodeling characteristics of contralateral ICA were related to CoW configurations.

https://thejns.org/doi/abs/10.3171/2017.5.JNS163112
\end{abstract}

KEY WORDS moyamoya disease; bypass; vessel remodeling; pressure drop; computational fluid dynamics; magnetic resonance angiography; vascular disorders

$\mathrm{M}$ OYAMOYa disease (MMD) is a progressive, occlusive disorder of the cerebral vasculature, with particular involvement of the circle of Willis (CoW). Outer-diameter narrowing of the involved arteries has been observed in patients with MMD. ${ }^{12}$ Intimal thickening is a typical pathological change, leading to stenosis and/or occlusion of the terminal portion of the internal carotid artery (ICA), as well as the middle and anterior cerebral arteries. As a result, the collateral ves- sels develop a hazy, film-like appearance, which is described as "moyamoya" on angiograms. MMD is thus regarded as a unique and dynamic cerebrovascular disease involving vascular flow from the IC to the external carotid (EC) system (which can develop transdural arterial recruitment), with the degree of involvement varying with the stages of MMD. ${ }^{6}$ Insufficiency of this IC-EC conversion system may result in cerebral ischemia, as well as intracranial hemorrhage from aneurysm development in

ABBREVIATIONS CFD = computational fluid dynamics; CoW = circle of Willis; $E C=$ external carotid; ICA = internal carotid artery; $\mathrm{MMD}=$ moyamoya disease; NOVA = Noninvasive Optimal Vessel Analysis; PD = pressure drop; PDI = PD index; QMRA = quantitative MR angiography; WSS = wall shear stress.

SUBMITTED December 13, 2016. ACCEPTED May 8, 2017.

INCLUDE WHEN CITING Published online October 20, 2017; DOI: 10.3171/2017.5.JNS163112. 
the collateral vascular networks, both of which contribute to the clinical presentation of MMD. Surgical revascularization by extracranial-intracranial bypass is thus the preferred procedure for treatment of MMD, complementing IC-EC conversion and thereby avoiding cerebral infarction and/or intracranial hemorrhage. ${ }^{6}$ Angiographic diminishment of moyamoya vessels, improvement in dilation of the anterior choroidal artery, and formation of collateral vascular networks from the EC system were observed following bypass surgery. ${ }^{10,11,15}$ However, the exact hemodynamic change of the IC system in MMD remains unknown.

One major view postulates the role of rising hemodynamic stress, caused by multiple stenotic changes in arteries at the base of the brain, in the development and progression of MMD. Reduction of hemodynamic stress on abnormal collaterals may be the core theoretical basis of revascularization treatment. ${ }^{9}$ Studies have yet to directly measure abnormalities in hemodynamic stress on the involved arteries. In our previous studies, we observed characteristic vessel remodeling in both the bypass graft and the ICA following bypass surgery. The changes in pressure drop (PD) along the ICA following bypass surgery were found to be a reliable hemodynamic parameter in the assessment of surgical treatment outcome. ${ }^{13,21,22}$

In this study, we assessed the morphological and hemodynamic modifications in the ICA following bypass surgery, through the use of quantitative MR angiography (QMRA) and computational fluid dynamics (CFD) technology, to aid in exploration of the mechanisms for revascularization in surgery for the treatment of MMD. Subgroup analysis was performed according to differing configurations of the $\mathrm{CoW}$.

\section{Methods \\ Patient Selection}

Following approval by the institutional review board, clinical data in all patients with MMD who underwent bypass surgeries at our institution between 2011 and 2012 were reviewed $(n=120)$. Among these patients, $68 \mathrm{had}$ undergone QMRA, and bilateral ICA flow rates were measured in 23 patients by QMRA, both before and after bypass surgery. These 23 patients were selected to undergo CFD simulation. All operations in this case series were performed unilaterally. MMD was diagnosed according to the criteria of the Research Committee on Spontaneous Occlusion of the Circle of Willis (Moyamoya Disease). ${ }^{7}$ This study was approved by the ethical committees. Written informed consent was obtained from each patient.

\section{Surgical Procedures}

Surgical intervention for MMD was indicated after comprehensive evaluations of angiography images, clinical manifestations, brain perfusion or metabolic findings, and detailed surgical indications as described previously. ${ }^{11,19}$ Anterior and/or posterior branches of the superficial temporal artery were anastomosed end-to-side to the cortical branch of the middle cerebral artery. Encephaloduro- myosynangiosis, the indirect bypass procedure involved, was described in our previous study. ${ }^{19}$

\section{Blood Flow and Vessel Diameter Measurements}

QMRA, which was implemented using Noninvasive Optimal Vessel Analysis (NOVA) software (VasSol, Inc.), was performed to measure the volumetric flow rate within the ICA. ${ }^{3,4}$ A 3D time-of-flight MRA scan (4 slabs, 40 slices per slab; TR $22 \mathrm{msec}$; TE $4.2 \mathrm{msec}$; flip angle $18^{\circ}$; matrix size $365 \times 384$; slice thickness $0.5 \mathrm{~mm}$; field of view $181 \times 200 \mathrm{~mm}^{2}$ ) was obtained with the 3.0-T MR Systems console (Verio, Siemens Medical Systems). Blood flow through the ICA was measured using commercially available NOVA software based on phase-contrast MRI, the accuracy of which has been validated both in vitro and in vivo. 5,20

\section{CFD Modeling}

3D geometries of the ICA were reconstructed and segmented through the use of MIMICS (Materialise' Interactive Medical Image Control System) to create domains for CFD computation. ${ }^{18,21}$ Mesh generation yielded elements ranging from 0.8 to 1.4 million. The conservation equations were solved through the use of ANSYS CFX 15.0, a finite volume-based CFD solver. Patient-specific inflow boundary conditions were measured using NOVA software based on MRA images. A zero static pressure was specified as an outlet boundary condition after extrusion of the outlet distally in a direction normal to the blood flow downstream to 30 times the size of the artery outlet. Blood flow was assumed as a laminar and Newtonian fluid with a density and dynamic viscosity of $1050 \mathrm{~kg} / \mathrm{m}^{-3}$ and $0.0032 \mathrm{~Pa} \cdot \mathrm{sec}$, respectively.

\section{PD Index}

The PD index (PDI) was calculated as the ratio of difference in pressure reduction changes along the ICA and preoperative pressure reduction. The PDI can be calculated using the formula

$$
\begin{aligned}
& \mathrm{PDI}=\Delta \mathrm{P}_{\mathrm{f}}-\Delta \mathrm{P}_{\mathrm{p}} / \Delta \mathrm{P}_{\mathrm{p}} \times 100 \%=\left[\left(\mathrm{P}_{\mathrm{if}}-\mathrm{P}_{\mathrm{of}}\right)-\right. \\
& \left.\left(\mathrm{P}_{\mathrm{ip}}-\mathrm{P}_{\mathrm{op}}\right)\right] / \Delta \mathrm{P}_{\mathrm{p}} \times 100 \%
\end{aligned}
$$

where $\Delta \mathrm{P}_{\mathrm{f}}$ and $\Delta \mathrm{P}_{\mathrm{p}}$ are the calculated pressure reduction at follow-up and preoperatively, respectively; $\mathrm{P}_{\text {if }}$ and $\mathrm{P}_{\text {of }}$ are the follow-up pressure values calculated at the inlet and outlet sections, respectively; and $\mathrm{P}_{\text {ip }}$ and $\mathrm{P}_{\text {op }}$ are the preoperative pressure values calculated at the ICA inlet and outlet, respectively. The positions of both proximal (inlet) and distal (outlet) planes, where we measured the pressure values, are the same between preoperative and follow-up models. The pressure was measured in $\mathrm{mm} \mathrm{Hg}$. The mean wall shear stress (WSS) of ICA was calculated using formula (2), with the WSS measured in $\mathrm{dyn} / \mathrm{cm}^{2}$

$$
\mathrm{WSS}=10 \mu(\Delta \mathrm{V} / \Delta \mathrm{y})
$$

where $\mu(\mathrm{Pa} \cdot \mathrm{sec})$ is flow viscosity, $\Delta \mathrm{V}(\mathrm{m} / \mathrm{sec})$ is the velocity difference at the near vessel wall surface, and $\Delta y$ $(\mathrm{m})$ is the distance to the vessel surface. 


\section{Statistical Analysis}

A 2-tailed, paired Student t-test was applied to compare the results of preoperative and follow-up parameters, both on the surgical side (surgical ICA) and the contralateral (nonsurgical) side (contralateral ICA) of the ICA. Regression analysis was used to assess the relationships between vessel diameter, PD, mean WSS, and volumetric flows before and after treatment. Statistical analysis was performed using SPSS version 17.0 software (IBM Corp.). Throughout the analysis, a significance level of $p \leq 0.05$ was assumed.

\section{Results}

\section{Patient Characteristics}

Ten male and 13 female patients with MMD were selected for this study. The mean age of patients was 35.3 \pm 9.2 years, ranging from 16 to 50 years. Postoperative follow-up and observations were conducted for 3-11 months, with an average follow-up of $6.5 \pm 3.1$ months. Patient characteristics and subtypes of MMD are outlined in Table 1 . All cases were classified into 2 groups according to the morphology of the CoW (with or without an intact arterial circle in bilateral ICAs) following analysis of the angiographic images: Group A (14 patients) had an incomplete CoW, and Group B (9 patients) had a complete $\mathrm{CoW}$. There were no significant differences in baseline hemodynamic values between surgical and contralateral ICAs prior to surgery, including vessel diameter, volumetric flow, PD, or WSS.

\section{Pressure Drop}

The mean PD ( \pm standard deviation) in surgical ICAs was $6.5 \pm 2.8 \mathrm{~mm} \mathrm{Hg}$ before surgery, compared with that in contralateral ICAs $(4.5 \pm 2.9 \mathrm{~mm} \mathrm{Hg} ; \mathrm{p}=0.09)$. For all cases, the mean PDI ( \pm standard deviation) of surgical ICAs was $21.18 \% \pm 30.1 \%$ ), compared with that of contralateral ICAs $(-11.75 \% \pm 28.6 \% ; p=0.001)$. When analyzed by group, the difference in PDI between surgical and contralateral ICAs was significant in Groups A and $\mathrm{B}(\mathrm{p}=0.002$ and $\mathrm{p}=0.04$, respectively). The mean PDIs of the surgical ICA in Groups A and B were $20.75 \%$ and $21.86 \%$, respectively $(p=0.934)$. However, the mean PDIs of the contralateral ICA in Groups A and B were 20.88\% and $2.45 \%$, respectively. This difference was significant ( $\mathrm{p}$ $=0.05)$ (Fig. 1).

Of these 23 patients, 15 underwent midterm follow-up ( $<6$ months), and 8 underwent long-term follow-up ( $>6$ months). Interestingly, the mean PDI ( \pm standard deviation) of the surgical ICA in the group with short-term follow-up was $17.7 \% \pm 8.3 \%$, a significantly higher value compared with the PDI of the contralateral ICA of $-17.3 \%$ $\pm 5.8 \%(\mathrm{p}=0.002)$. The PDIs of the surgical and contralateral ICA in the long-term follow-up group, however, were $25.6 \% \pm 8.3 \%$ and $-1.2 \% \pm 13.1 \%$, respectively $(\mathrm{p}=0.11)$ (Supplemental Fig. 1).

\section{Volumetric Flow Rate}

The mean volumetric flow rate ( \pm standard deviation) in the surgical ICA decreased significantly from $159.65 \pm$
TABLE 1. Summary of patient characteristics, MMD features, surgical procedures, and baseline hemodynamic values

\begin{tabular}{|c|c|}
\hline Variable & Value \\
\hline No. of patients & 23 \\
\hline Female, $\%$ & 56.5 \\
\hline Mean age, yrs (range) & $35.3(16-50)$ \\
\hline Ischemic presentation, $\%$ & 65.2 \\
\hline \multicolumn{2}{|l|}{ MMD characteristics } \\
\hline \multicolumn{2}{|l|}{ Suzuki stage, no. of hemispheres } \\
\hline 1 & 0 \\
\hline II & 4 \\
\hline III & 22 \\
\hline IV & 14 \\
\hline V & 6 \\
\hline $\mathrm{VI}$ & 0 \\
\hline \multicolumn{2}{|l|}{ Surgery, no. (\%) } \\
\hline Lt side & $14(60.9)$ \\
\hline Single anastomosis & $20(87.0)$ \\
\hline Encephaloduromyosynangiosis & $23(100)$ \\
\hline \multicolumn{2}{|l|}{$\begin{array}{l}\text { Morphology \& hemodynamic baseline } \\
\text { values, mean } \pm \text { SD }\end{array}$} \\
\hline \multicolumn{2}{|l|}{ Vessel diameter, $\mathrm{mm}$} \\
\hline Surgical ICA & $3.44 \pm 0.84$ \\
\hline Contralat ICA & $3.59 \pm 0.96$ \\
\hline \multicolumn{2}{|l|}{ ICA flow, $\mathrm{ml} / \mathrm{min}$} \\
\hline Surgical ICA & $159.65 \pm 71.48$ \\
\hline Contralat ICA & $165.20 \pm 51.96$ \\
\hline \multicolumn{2}{|l|}{$\mathrm{PD}, \mathrm{mm} \mathrm{Hg}$} \\
\hline Surgical ICA & $6.5 \pm 2.8$ \\
\hline Contralat ICA & $4.5 \pm 2.9$ \\
\hline \multicolumn{2}{|l|}{ WSS, dyn/cm² } \\
\hline Surgical ICA & $77.0 \pm 28.1$ \\
\hline Contralat ICA & $64.3 \pm 37.8$ \\
\hline
\end{tabular}

$71.48 \mathrm{ml} / \mathrm{min}$ to $135.97 \pm 68.89 \mathrm{ml} / \mathrm{min}(\mathrm{p}=0.01)$. However, in the contralateral ICA, volumetric flow rates underwent an insignificant increase $(165.20 \pm 51.96 \mathrm{ml} / \mathrm{min}$ preoperatively vs $174.97 \pm 97.47 \mathrm{ml} / \mathrm{min}$ at follow-up; $\mathrm{p}$ $=0.46$ ). The volumetric flow rate in the surgical ICA decreased by $14.83 \%$ compared with that in the contralateral ICA, which increased by $0.96 \%(p=0.17)$. There were no significant differences in percentage changes for volumetric flow rates between surgical and contralateral ICAs in either Groups A or B (11.11\% vs $17.48 \%$ and $9.40 \%$ vs $-9.02 \%$, respectively).

\section{Wall Shear Stress}

At follow-up observation, the mean WSS ( \pm standard deviation) in the surgical ICA decreased significantly from $64.88 \pm 28.05 \mathrm{dyn} / \mathrm{cm}^{2}$ to $55.55 \pm 37.93 \mathrm{dyn} / \mathrm{cm}^{2}(\mathrm{p}$ $=0.05)$. In the contralateral ICA, however, the mean WSS also decreased significantly $\left(64.33 \pm 37.80 \mathrm{dyn} / \mathrm{cm}^{2}\right.$ preoperatively vs $49.94 \pm 17.61 \mathrm{dyn} / \mathrm{cm}^{2}$ at follow-up; $\mathrm{p}=0.01$ ). 


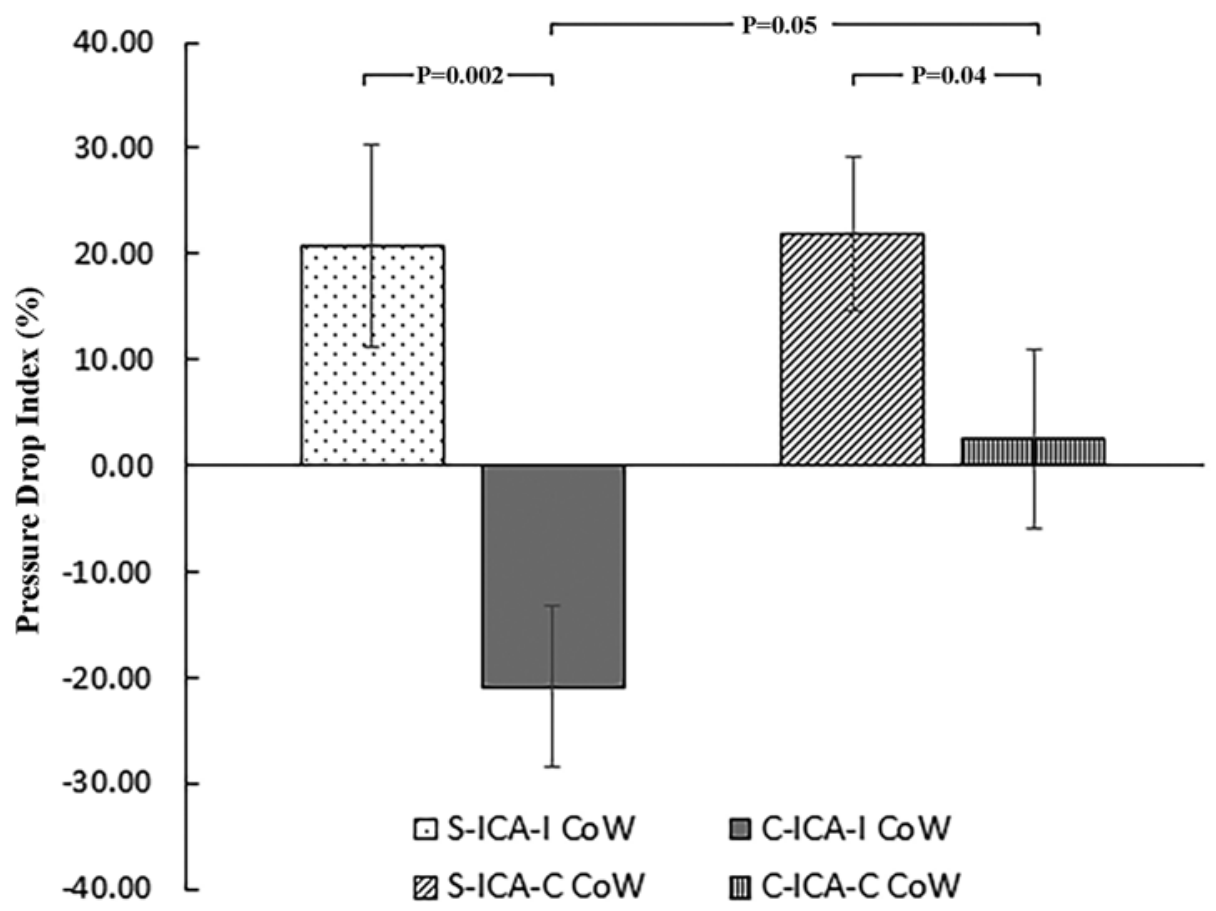

FIG 1. The difference in the PDI between groups with incomplete and complete CoW. The error bar represents the standard error. S-ICA-I CoW indicates surgical ICA with incomplete CoW; C-ICA-I CoW indicates contralateral ICA with incomplete CoW; S-ICA-C CoW indicates surgical ICA with complete CoW; C-ICA-C CoW indicates contralateral ICA with complete CoW.

The percentage change in the mean WSS in the surgical ICA was $10.67 \%$, which was not significantly different than the value found in the contralateral ICA $(12.41 \%$; $\mathrm{p}=$ 0.87). There were no significant differences in percentage changes in inflow rates between surgical and contralateral ICAs in Groups A and B (6.46\% vs $13.69 \%$ and $17.21 \%$ vs $10.56 \%$, respectively).

\section{Predictors of Decreased PD After Bypass Surgery}

Linear regression analysis revealed that neither the ages of patients $(p=0.12)$ nor Suzuki stages of MMD $(\mathrm{p}=0.54)$ were significantly associated with a decreased PD (Table 2). A greater decrease in PD postoperatively corresponded to greater increases in the mean WSS $\left(\mathrm{R}^{2}\right.$ $=0.23, p=0.02)$, decreases in volumetric flow $\left(R^{2}=\right.$ $0.51, \mathrm{p}=0.000$ ), and was significantly associated with a reduction in the luminal diameter of ICAs $\left(\mathrm{R}^{2}=0.22\right.$, $\mathrm{p}=0.02$ ) (Figs. 2 and 3, and Supplemental Fig. 2). Multivariate analysis, however, demonstrated that the decreases in volumetric flow $(\mathrm{p}=0.001)$, but not increases in WSS $(p=0.14)$ nor reductions in diameter $(p=0.29)$, remained predictive of decreased PD in ICAs after surgery (Table 2).

\section{Discussion}

Several studies have demonstrated that bypass surgery can prevent ischemic and hemorrhagic stroke in patients with MMD by improving cerebral blood flow through ischemic brain via newly constructed vessel networks from the EC system, thereby lowering the hemodynamic stress of an abnormal IC system. ${ }^{4,8,15}$ Improvements in anterior choroidal artery dilation and decreases in moyamoya vessels have been hypothesized to be relevant angiographic changes following bypass. ${ }^{11,15}$ Most researchers, however, focus on morphometric analysis of vessels through angiographic and clinical data, with little research done to analyze hemodynamic mechanisms. CFD may contribute to a greater understanding of hemodynamic stress, because it has been used to quantify blood flow patterns and PDs in aneurysms and their arteries of origin. ${ }^{16}$

In this study, CFD technology was used to measure the PD along the ICA pre- and postoperatively. In addition, the mean WSS and flow rate through the ICA were quantified. Flow rates and PDs in the surgical ICA declined significantly following surgery (Fig. 4). Flow rate in surgical ICAs decreased by $14.83 \%$, while the PD decreased by $21.18 \%$. This phenomenon correlates with the theory that MMD is a cerebrovascular disease underpinned by the IC-EC conversion system, ${ }^{6}$ with bypass surgery thereby

TABLE 2. Predictors of decreased PD in the ICA after surgery

\begin{tabular}{lcccc}
\hline \multirow{2}{*}{ Variable } & \multicolumn{2}{c}{$\begin{array}{c}\text { Univariate } \\
\text { Analysis }\end{array}$} & & $\begin{array}{c}\text { Multivariate } \\
\text { Analysis }\end{array}$ \\
\cline { 2 - 3 } \cline { 5 - 5 } & R Value & $p$ Value & & $p$ Value \\
\hline Decreases in diameter & -0.467 & 0.025 & & 0.288 \\
\hline Increases in mean WSS & 0.474 & 0.022 & & 0.144 \\
\hline Decreases in volumetric flow & 0.712 & 0.000 & & 0.001 \\
\hline
\end{tabular}




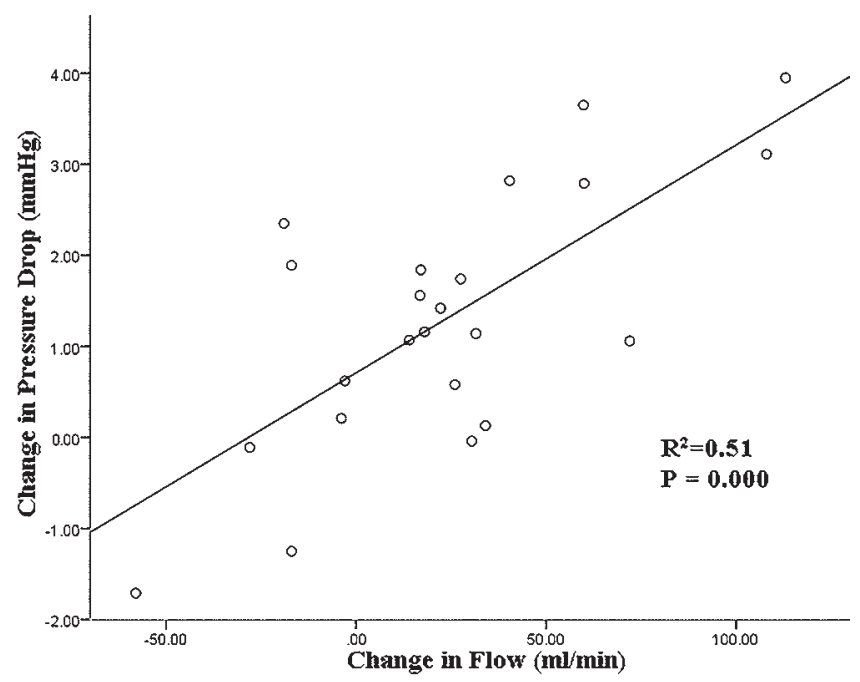

FIG 2. Changes in volumetric flow versus PD after surgery. A greater decrease in $\mathrm{PD}$ postoperatively corresponded to a greater reduction in volumetric flow $\left(R^{2}=0.51, p=0.000\right)$.

useful in helping to complete the process of IC-EC conversion. After new collaterals are built, the hemodynamic function of the ICA may be weakened, with reduced hemodynamic stress following functional revascularization surgery.

The CoW is an important structure in creating redundancies or collaterals in cerebral circulation. ${ }^{2}$ The PDI in the contralateral ICA in the group with complete CoW decreased by $2.45 \%$ at follow-up, whereas the PDI in the group with incomplete $\mathrm{CoW}$ increased by $20.88 \%$. This may have been due to blood flow into the contralateral ICA through an intact arterial circle in bilateral ICAs. PD in the surgical ICA following bypass surgery may be matched to the contralateral ICA due to the connection of bilateral ICAs through the CoW.

The WSS represents the force of blood against the vessel wall, which is applied mainly to the endothelium. This hemodynamic force has been identified as a critical determinant of vessel diameter and vascular remodeling through the proliferation of endothelial progenitor cells. ${ }^{16}$ In addition, the WSS is also regarded as an important physical force that is vital to inducing the remodeling of preexisting arterioarteriolar anastomoses, or arteriogenesis, which may contribute to the mechanisms underlying bypass surgery for treatment of MMD. ${ }^{17}$ More recently, Alaraj et al. demonstrated that WSS was a potential biomechanical factor for efficient treatment of arteriovenous malformations. ${ }^{1}$ In the present study, the mean WSS of both surgical and nonsurgical ICAs decreased, with the difference in mean WSS between surgical and contralateral ICAs following surgery found to be not significant. Because multiple stenotic changes occur along the ICA, the mean WSS may not be a sensitive parameter in the measurement of hemodynamic changes following surgery for treatment of MMD.

Overall, our findings clarify the hemodynamics underlying MMD and quantify the effects of bypass surgery.

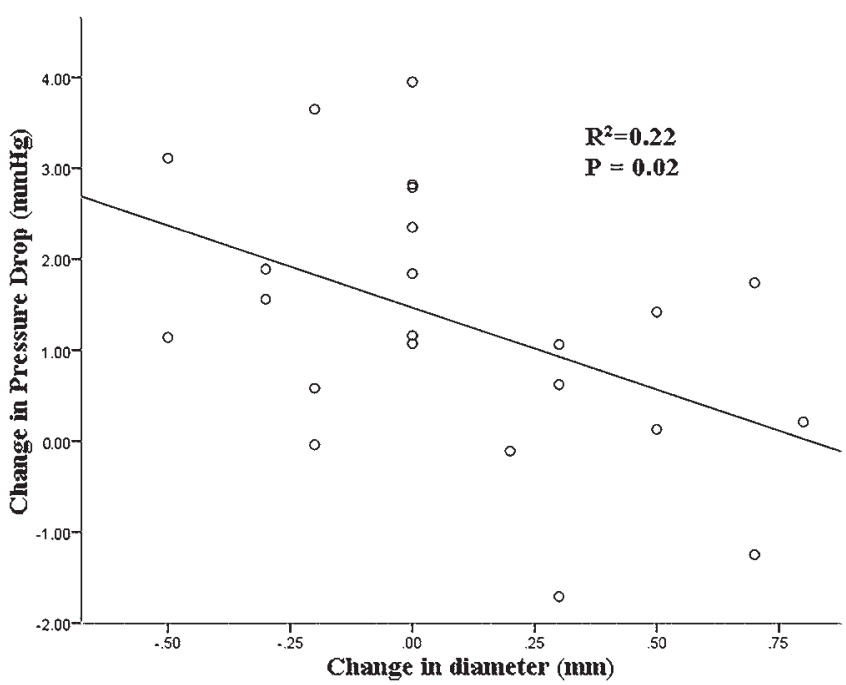

FIG 3. Changes in vessel diameter versus PD after surgery. A greater decrease in PD postoperatively corresponded to the shrinking of ICAs $\left(R^{2}=0.22, p=0.02\right)$.

Findings from this study indicate that vessel remodeling occurs in surgical ICAs following bypass surgery, which is associated with reductions in vessel diameter, volumetric flow, PD, and mean WSS. This pattern of vessel remodeling correlates with the dynamic nature of MMD, otherwise termed IC-EC conversion. From a hemodynamic perspective, bypass surgery has been proven to help complete the IC-EC conversion process. More specifically, the PDI has been identified as a potential biomechanical factor that may be important in remodeling of the ICA over time and in the decline in hemodynamic stress in the IC system following bypass surgery. Configurations of the CoW will likewise influence the effect of bypass surgery on the contralateral ICA.

Possible limitations of this study include its retrospective design and small sample size. However, to our knowledge, this is currently the largest study cohort for hemodynamic analysis following bypass surgery in patients with MMD, through the use of patient-specific QMRA assessment and CFD technology. Moreover, exact flow measurements using QMRA ensured greater accuracy of CFD simulation results. Another plausible shortcoming of this study is that the follow-up may not have been long enough to monitor the vessel remodeling processes in the contralateral ICA, because MMD is a gradually progressive cerebrovascular disorder, with a mean period of progression of 12-34 months. ${ }^{14}$

\section{Conclusions}

Our results confirm the occurrence of IC-EC conversion following bypass surgery, indicating the role of vessel remodeling in the ICA after bypass surgery in reducing PD and associated decreases in vessel diameter and volumetric flow. Moreover, configurations of the CoW will influence the effect of bypass surgery on the contralateral ICA. CFD may be a useful tool for virtually estimating 

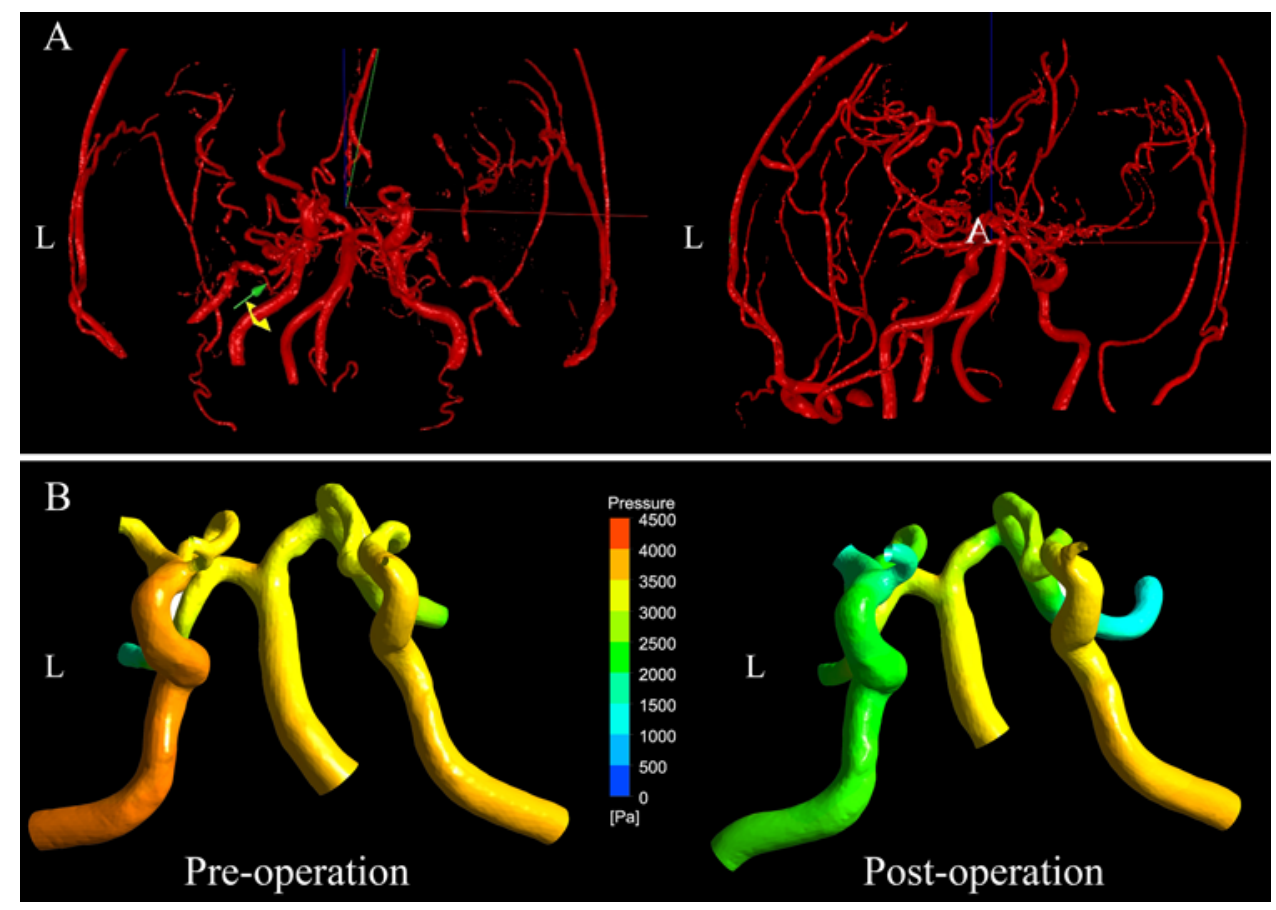

FIG 4. Illustrative case. A: Pre- and postoperative QMRA images showing the effects of bypass surgery in the left hemisphere (L). The volumetric flow of the ICA decreased from $145 \mathrm{ml} / \mathrm{min}$ to $110 \mathrm{ml} / \mathrm{min}$, with more than two-thirds of the middle cerebral artery area of distribution supplied by newly constructed vessel networks from the EC system. On left panel, arrows indicate direction of the flow; on right panel, A indicates anterior view. B: Corresponding pressure contours showing that the PD decreased in bilateral ICAs.

the outcome of bypass surgery for treatment of MMD. Continuous long-term hemodynamic monitoring is recommended for the contralateral hemisphere in the event of disease progression.

\section{Acknowledgments}

This study was sponsored by Australian Research Council Discovery Project (DP1112985) (Qian, Morgan), National Key Technology R\&D Program of the Ministry of Science and Technology of China (no. 2011BAI08B06) (Mao), Key Medical Project of Shanghai Science and Technology Commission (no. 13411950200) (Mao), The Foundation of National Natural Science Fund, China (no. 81401136 and 81171082) (Zhu, Mao), and Project of Shanghai Science and Technology Commission (no. 16495800300) (Tian).

\section{References}

1. Alaraj A, Shakur SF, Amin-Hanjani S, Mostafa H, Khan S, Aletich VA, et al: Changes in wall shear stress of cerebral arteriovenous malformation feeder arteries after embolization and surgery. Stroke 46:1216-1220, 2015

2. Alnaes MS, Isaksen J, Mardal KA, Romner B, Morgan MK, Ingebrigtsen T: Computation of hemodynamics in the circle of Willis. Stroke 38:2500-2505, 2007

3. Amin-Hanjani S, Shin JH, Zhao M, Du X, Charbel FT: Evaluation of extracranial-intracranial bypass using quantitative magnetic resonance angiography. J Neurosurg 106:291-298, 2007

4. Amin-Hanjani S, Singh A, Rifai H, Thulborn KR, Alaraj A, Aletich V, et al: Combined direct and indirect bypass for moyamoya: quantitative assessment of direct bypass flow over time. Neurosurgery 73:962-968, 2013
5. Calderon-Arnulphi M, Amin-Hanjani S, Alaraj A, Zhao M, Du X, Ruland S, et al: In vivo evaluation of quantitative MR angiography in a canine carotid artery stenosis model. AJNR Am J Neuroradiol 32:1552-1559, 2011

6. Fujimura M, Tominaga T: Current status of revascularization surgery for Moyamoya disease: special consideration for its 'internal carotid-external carotid (IC-EC) conversion' as the physiological reorganization system. Tohoku J Exp Med 236:45-53, 2015

7. Fukui M: Guidelines for the diagnosis and treatment of spontaneous occlusion of the circle of Willis ('moyamoya' disease). Research Committee on Spontaneous Occlusion of the Circle of Willis (Moyamoya Disease) of the Ministry of Health and Welfare, Japan. Clin Neurol Neurosurg 99 (Suppl 2):S238-S240, 1997

8. Hoshino T, Yokose N, Awano T, Nakamura S, Fujiwara N, Murata Y, et al: Effects of EC-IC bypass surgery on cerebral perfusion in moyamoya disease and non-moyamoya ischemic stroke evaluated by optical techniques. J Cereb Blood Flow Metab 27 (1 Suppl):BO01-07, 2007 (Abstract)

9. Houkin K, Kamiyama H, Abe H, Takahashi A, Kuroda S: Surgical therapy for adult moyamoya disease. Can surgical revascularization prevent the recurrence of intracerebral hemorrhage? Stroke 27:1342-1346, 1996

10. Houkin K, Nakayama N, Kuroda S, Ishikawa T, Nonaka T: How does angiogenesis develop in pediatric moyamoya disease after surgery? A prospective study with MR angiography. Childs Nerv Syst 20:734-741, 2004

11. Jiang H, Ni W, Xu B, Lei Y, Tian Y, Xu F, et al: Outcome in adult patients with hemorrhagic moyamoya disease after combined extracranial-intracranial bypass. J Neurosurg 121:1048-1055, 2014

12. Kaku Y, Morioka M, Ohmori Y, Kawano T, Kai Y, Fukuoka $\mathrm{H}$, et al: Outer-diameter narrowing of the internal carotid 
and middle cerebral arteries in moyamoya disease detected on 3D constructive interference in steady-state MR image: is arterial constrictive remodeling a major pathogenesis? Acta Neurochir (Wien) 154:2151-2157, 2012

13. Karunanithi K, Han C, Lee CJ, Shi W, Duan L, Qian Y: Identification of a hemodynamic parameter for assessing treatment outcome of EDAS in Moyamoya disease. J Biomech 48:304-309, 2015

14. Kelly ME, Bell-Stephens TE, Marks MP, Do HM, Steinberg GK: Progression of unilateral moyamoya disease: A clinical series. Cerebrovasc Dis 22:109-115, 2006

15. Miyamoto S, Yoshimoto T, Hashimoto N, Okada Y, Tsuji I, Tominaga T, et al: Effects of extracranial-intracranial bypass for patients with hemorrhagic moyamoya disease: results of the Japan Adult Moyamoya Trial. Stroke 45:1415-1421, 2014

16. Papaioannou TG, Stefanadis C: Vascular wall shear stress: basic principles and methods. Hellenic J Cardiol 46:9-15, 2005

17. Saito N, Imai H: Insights on the revascularization mechanism for treatment of moyamoya disease based on the histopathologic concept of angiogenesis and arteriogenesis. World Neurosurg 75:204-205, 2011

18. Sia SF, Qian Y, Zhang Y, Morgan MK: Mean arterial pressure required for maintaining patency of extracranial-to-intracranial bypass grafts: an investigation with computational hemodynamic models-case series. Neurosurgery 71:826831,2012

19. Xu B, Song DL, Mao Y, Gu YX, Xu H, Liao YJ, et al: Superficial temporal artery-middle cerebral artery bypass combined with encephalo-duro-myo-synangiosis in treating moyamoya disease: surgical techniques, indications and midterm follow-up results. Chin Med J (Engl) 125:4398-4405, 2012

20. Zhao M, Charbel FT, Alperin N, Loth F, Clark ME: Improved phase-contrast flow quantification by three-dimensional vessel localization. Magn Reson Imaging 18:697706, 2000

21. Zhu F, Karunanithi K, Qian Y, Mao Y, Xu B, Gu Y, et al: Assessing surgical treatment outcome following superficial temporal artery to middle cerebral artery bypass based on computational haemodynamic analysis. J Biomech 48:40534058, 2015

22. Zhu FP, Zhang Y, Higurashi M, Xu B, Gu YX, Mao Y, et al: Haemodynamic analysis of vessel remodelling in STA-MCA bypass for Moyamoya disease and its impact on bypass patency. J Biomech 47:1800-1805, 2014

\section{Disclosures}

The authors report no conflict of interest concerning the materials or methods used in this study or the findings specified in this paper.

\section{Author Contributions}

Conception and design: Qian, F Zhu, Xu, Gu, Mao, Morgan. Acquisition of data: Qian, F Zhu, Gu, Karunanithi, W Zhu, Chen, Mao. Analysis and interpretation of data: Qian, F Zhu, Xu, Karunanithi, W Zhu, Morgan. Drafting the article: Qian, F Zhu, Xu, Gu, W Zhu, Chen, Mao. Critically revising the article: Qian, F Zhu, Mao. Reviewed submitted version of manuscript: Qian, F Zhu, Karunanithi, Chen, Mao, Morgan. Approved the final version of the manuscript on behalf of all authors: Qian. Statistical analysis: Qian, F Zhu. Administrative/technical/material support: Qian, Mao. Study supervision: Qian, Mao, Morgan.

\section{Supplemental Information Online-Only Content}

Supplemental material is available with the online version of the article.

Supplemental Figures 1 and 2. https://thejns.org/doi/suppl/ 10.3171/2017.5.JNS163112.

\section{Correspondence}

Yi Qian, Faculty of Medicine and Health Sciences, Macquarie University, 2 Technology Pl., Sydney, NSW 2109, Australia. email: yi.qian@mq.edu.au. 